\title{
Stretching positions for the coracohumeral ligament: Strain measurement during passive motion using fresh/frozen cadaver shoulders
}

\author{
Tomoki Izumi ${ }^{{ }^{*}+}$, Mitsuhiro Aoki ${ }^{2 \dagger}$, Yoshitaka Tanaka $^{3 \dagger}$, Eiichi Uchiyama ${ }^{4}$, Daisuke Suzuki ${ }^{5}$, Shigenori Miyamoto ${ }^{6}$, \\ Mineko Fujimiya ${ }^{5}$
}

\begin{abstract}
Background: Contracture of the coracohumeral ligament is reported to restrict external rotation of the shoulder with arm at the side and restrict posterior-inferior shift of the humeral head. The contracture is supposed to restrict range of motion of the glenohumeral joint.

Methods: To obtain stretching position of the coracohumeral ligament, strain on the ligament was measured at the superficial fibers of the ligament using 9 fresh/frozen cadaver shoulders. By sequential measurement using a strain gauge, the ligament strain was measured from reference length (LO). Shoulder positions were determined using a 3 Space Tracker System. Through a combination of previously reported coracohumeral stretching positions and those observed in preliminary measurement, ligament strain were measured by passive external rotation from $10^{\circ}$ internal rotation, by adding each $10^{\circ}$ external rotation, to maximal external rotation.

Results: Stretching positions in which significantly larger strain were obtained compared to the L0 values were $0^{\circ}$ elevation in scapula plane with $40^{\circ}, 50^{\circ}$ and maximum external rotation $(5.68 \%, 7.2 \%, 7.87 \%), 30^{\circ}$ extension with $50^{\circ}$, maximum external rotation $(4.20 \%, 4.79 \%)$, and $30^{\circ}$ extension + adduction with $30^{\circ}, 40^{\circ}, 50^{\circ}$ and maximum external rotation $(4.09 \%, 4.67 \%, 4.78 \%, 5.05 \%)(\mathrm{P}<0.05)$. No positive strain on the coracohumeral ligament was observed for the previously reported stretching positions; ie, $90^{\circ}$ abduction with external rotation or flexion with external rotation.
\end{abstract}

Conclusions: Significant strain of the coracohumeral ligament will be achieved by passive external rotation at lower shoulder elevations, extension, and extension with adduction.

\section{Background}

External rotation of the glenohumeral joint is important to maintain activity of daily living and it contributes to allow flexion and abduction of the shoulder joint. [1] Patients suffering a frozen shoulder or rotator cuff tear demonstrate restriction of abduction and external rotation of the glenohumeral joint. $[1,2]$ For such cases, physiotherapy may help to stretch the soft tissue and maintain mobility of the glenohumeral joint. [3]

Harryman et al [4] reported that external rotation of the glenohumeral joint at 0 degrees of elevation was

\footnotetext{
* Correspondence: t.izumi@sapmed.ac.jp

+ Contributed equally

'Doctoral Course of Physical Therapy, Graduate School of Health Sciences,

Sapporo Medical University, Sapporo, Japan

Full list of author information is available at the end of the article
}

reduced by approximately $50 \%$ in patients with contracture of the rotator interval involving the coracohumeral ligament. Previous authors have also reported that contracture of the rotator interval reduced external rotation at 0 degrees of elevation, $[2,5]$ and external rotation in flexion and abduction. [4,6,7] Improvement of abduction and flexion of the glenohumeral joint was obtained by surgical release of the coracohumeral ligament in patients who had severe contracture of the joint. $[5,7]$ Neer et al [5] and Gagey et al [8] reported that the coracohumeral ligament played a role in limiting the range of motion for external rotation of the glenohumeral joint. Bowen et al [9] and Warner et al [10], however, reported that the ligament acted to limit the inferior shift of the humeral head, whereas, Harryman et al [4] 
reported that the ligament limited the posterior shift of the humeral head.

In physiotherapy, it has been experienced that elongation of the coracohumeral ligament in the rotator interval can provide an increase in the range of external rotation and flexion of the glenohumeral joint. Thus, we consider that non-invasive stretching of the coracohumeral ligament may not only improve the range of motion of the glenohumeral joint but reduce pain due to contracture of the joint.

Various stretching procedures for the coracohumeral ligament have been reported in literature; however, no consensus has been reached [11-16]. These stretching procedures have been obtained from observations of the anatomical location and length of the ligament and capsule, and have been used as a standard in physiotherapy. However, they were not verified by the measurement of the strain. Therefore, it is important to measure the strain on the coracohumeral ligament to disclose appropriate stretching positions for the coracohumeral ligament. [11,13-17]

In this study, nine fresh/frozen cadaver shoulders were used to perform strain measurement and determine stretch positions for the coracohumeral ligament. Strain on the coracohumeral ligament was measured during passive external rotation at nine designated shoulder positions, in which the reference length (L0) of the ligaments was taken into consideration. The purpose of this study is to indicate procedures for potential stretch positions for the coracohumeral ligament in vivo.

\section{Materials and methods \\ Preparation and Specimens}

Nine fresh/frozen shoulder specimens (6 males, 3 females) without evidence of tendon rupture or osteoarthritis were used in this experiment. In this study, we observed articular cartilage of the humeral head and glenoid fossa of the scapula after the study, and specimens with osteophytes or abrasion of the articular cartilage were excluded. [18] The age of specimens at death ranged from 79 to 96 years (mean, 86.3 years). Within 24. hours after death, the specimens were transferred from regional hospitals to the $2^{\text {nd }}$ Department of Anatomy of our university. Shoulder specimens were disarticulated from the thorax and kept in a freezer at $-20^{\circ} \mathrm{C}$. Thawing of the shoulder specimens at room temperature $\left(22^{\circ} \mathrm{C}\right)$ was started 12 hours before preparation.

The skin, fascia, muscles, nerves, and vessels were removed sparing the rotator cuff and coracohumeral ligament. In addition, the coracoacromial ligament and anterior part of the acromion were removed, and the coracohumeral ligament and tendon of supraspinatus were exposed. The distal third of the humerus was exposed, and an acrylic stick was inserted perpendicular to the humerus shaft, indicating the direction of the forearm. Next, the humerus was amputated above the elbow. During the experiment, the specimens were kept moist by spraying with saline every 5 to 10 minutes. Room temperature was maintained at $22^{\circ} \mathrm{C}$.

\section{Testing Apparatus}

A wooden jig, consisting of a wooden board and a square post/column (height $500 \mathrm{~mm} \times$ width $160 \mathrm{~mm} \times$ thickness $24 \mathrm{~mm}$ ), was used for this experiment. The ventral surface of the scapula of the specimen was fixed to the wooden post/column so that the medial border of the scapula was perpendicular to the ground to simulate the resting scapular position (Figure 1). Two anchors (Fastin RC threaded suture anchor, Mitek, Massachusetts, USA) were inserted into the bony insertion of the subscapularis tendon and infraspinatus tendon to apply a compression force of $11 \mathrm{~N}$ (total $22 \mathrm{~N}$ ) via a suture against the glenoid fossa. In previous cadaveric studies, this compression force was used as the minimum force required to prevent subluxation of the humeral head from the glenoid fossa during passive glenohumeral motion. [4,19-22] In this study, humeral head subluxation was carefully observed by three researchers and no visible and palpable subluxation was detected during experiment. A thin wooden rod (400 $\mathrm{mm}$ in length) was then inserted into the medullary canal of the humerus to help in maintaining glenohumeral elevation, abduction, flexion, and extension at the designated angle during passive external rotation of the humerus.

\section{Measurement device}

The strain data for the coracohumeral ligament was obtained from a displacement sensor (Pulse Coder,

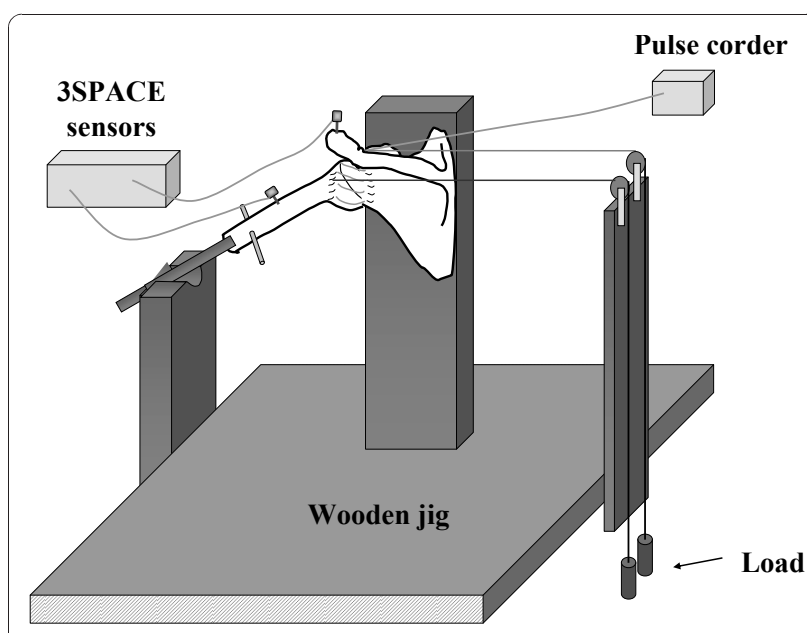

Figure 1 Experimental set-up. A displacement sensor was attached to the superficial fibers of the coracohumeral ligament. A six-degree of freedom electromagnetic tracking device was used to monitor the glenohumeral rotational angles. 
LEVEX, Kyoto, Japan). The linearity error and repetitive accuracy of the Pulse Coder are below $1 \%$ and $2.5 \mu \mathrm{m}$, respectively. The stroke of the Pulse Coder at this linearity is $14 \mathrm{~mm}$, and all strain measurements were performed within this stroke range. The Pulse Coder consisted of coil sensor and a brass pipe, in which the rod of the coil sensor located. In previous mechanical studies, strain on the ligament and tendon were measured using DVRT (Differential Variable Reluctance Transducer, Microstrain, Williston, Vermont). The mass effect of DVRT was not described in previous reports; [19-21,23,24] however, it can be neglected as the weight of the Pulse Corder is $10.16 \mathrm{~g}$. The sensors were attached to the center of the superficial fiber of the coracohumeral ligament, $[11,25,26]$ and were placed parallel to the ligament fibers (Figure 2).

A six-degree of freedom electromagnetic tracking device (3SPACE FASTRACK, Polhemus, Colchester, Vermont) was used to monitor the glenohumeral angles during measurement. [27] The length, width, height and weight of the Polhemus Sensors were $2.3 \mathrm{~cm}, 2.8 \mathrm{~cm}$, $1.5 \mathrm{~cm}$ and $17 \mathrm{~g}$, respectively. [27] Polhemus sensors were fixed on the bone with titanic screws. This form of fixation is rigid. Stability of the sensor was observed in the monitor. The angle of the glenohumeral joint was simulated by the angle between the plane of the glenoid fossa and the longitudinal line of the humerus. Rotational angle was simulated by rotation of humerus along its longitudinal axis [28]. The glenoid fossa tilts 4 degrees superiorly toward the medial border of the scapula, and at 7 degrees of retroversion [28]. As the scapula of the specimen was fixed on the wooden jig, adjusting the anterior surface of the scapular so as to be parallel to the frontal plane, allows the plane of the glenoid fossa to be determined on the basis of anatomical knowledge. This device enabled measurement of the

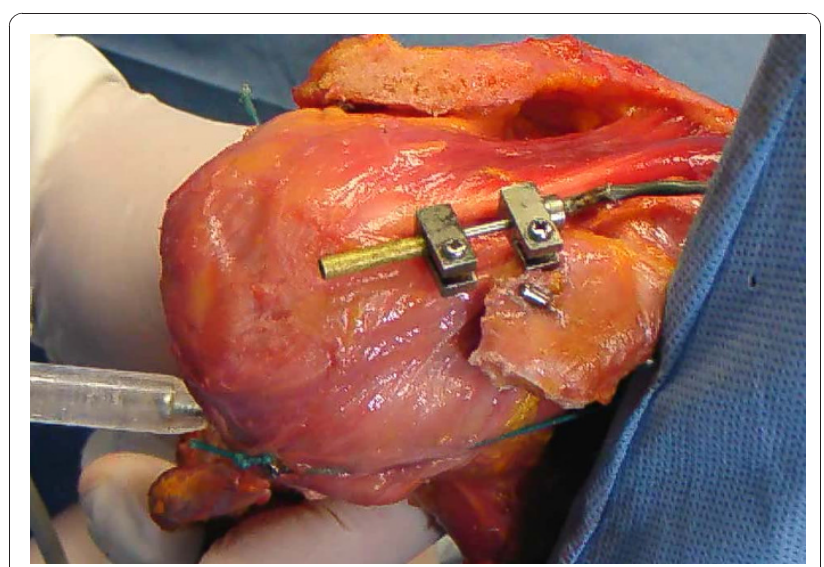

Figure $2 \mathrm{~A}$ photograph of the displacement sensor. The sensor was attached to the center of the superficial fibers of the coracohumeral ligament parallel to the ligament fibers. three-dimensional position and orientation of the sensors relative to the absolute coordinates generated by the source. One sensor was placed on the acromion and the other was placed on the middle portion of the humerus. In this system, angle of the arm flexion, abduction and extension was defined as the angle between the plane of the glenoid fossa and the longitudinal axis of the humerus. The rotation angle was defined as the rotation of the humerus along its longitudinal axis. Within a $750 \mathrm{~mm}$ range from the source, the positional accuracy was $0.8 \mathrm{~mm}$ root-mean-square, and the angular accuracy was $0.5^{\circ}$ root-mean-square. [27]

\section{Experimental procedure Measurement portion}

The coracohumeral ligament is reported to run from the base of the coracoid process to the greater and lesser tuberosities of the humerus. [11] The coracohumeral ligament is classified into superficial and deep fibers, the former inserts into the greater tuberosity and the latter inserts into the lesser tuberosity. [25,26] In this experiment, strain on the superficial fibers, but not the deep fibers, of the coracohumeral ligament was measured, because superficial fibers are considered to from the major part of this ligament. [12] In this study, strain was measured on the central superficial fibers of the coracohumeral ligament as Bigliani et al reported that tensile properties were measured at the center of the inferior glenohumeral ligament, [29] and Noyes et al [30] reported that tensile properties were the highest at the center of the patellar tendon, and those values were used to represent the whole patella tendon. (Figure 2)

\section{Measurement positions}

To measure strain on the coracohumeral ligament, measurement positions were designated by a combination of positions that have been previously reported in literature and those obtained from our preliminary experiments. Based on the anatomic position of the coracohumeral ligament, which located on the antero-superior aspect of the glenohumeral joint, passive stretching by external rotation was applied to the specimens in each of the designated positions. Range of passive external rotation of the specimens used in this study was from $-10^{\circ}$ to maximal rotation. In each shoulder position, passive external rotation in $10^{\circ}$ increments was applied (Figure 3: Globe-graph of clinical positioning).

In this study, we defined each joint movement using the Globe system. [19-21,31] The scapula plane was set at a longitude of 0 degrees in the Globe system. The ventral and dorsal aspects of the scapula were defined as positive and negative values, respectively, as shown in Figure 3. Further, 0 degrees elevation of the humerus was set at 0 degrees of latitude, with maximum elevation at 180 degrees of latitude. The scapula is protracted 30 

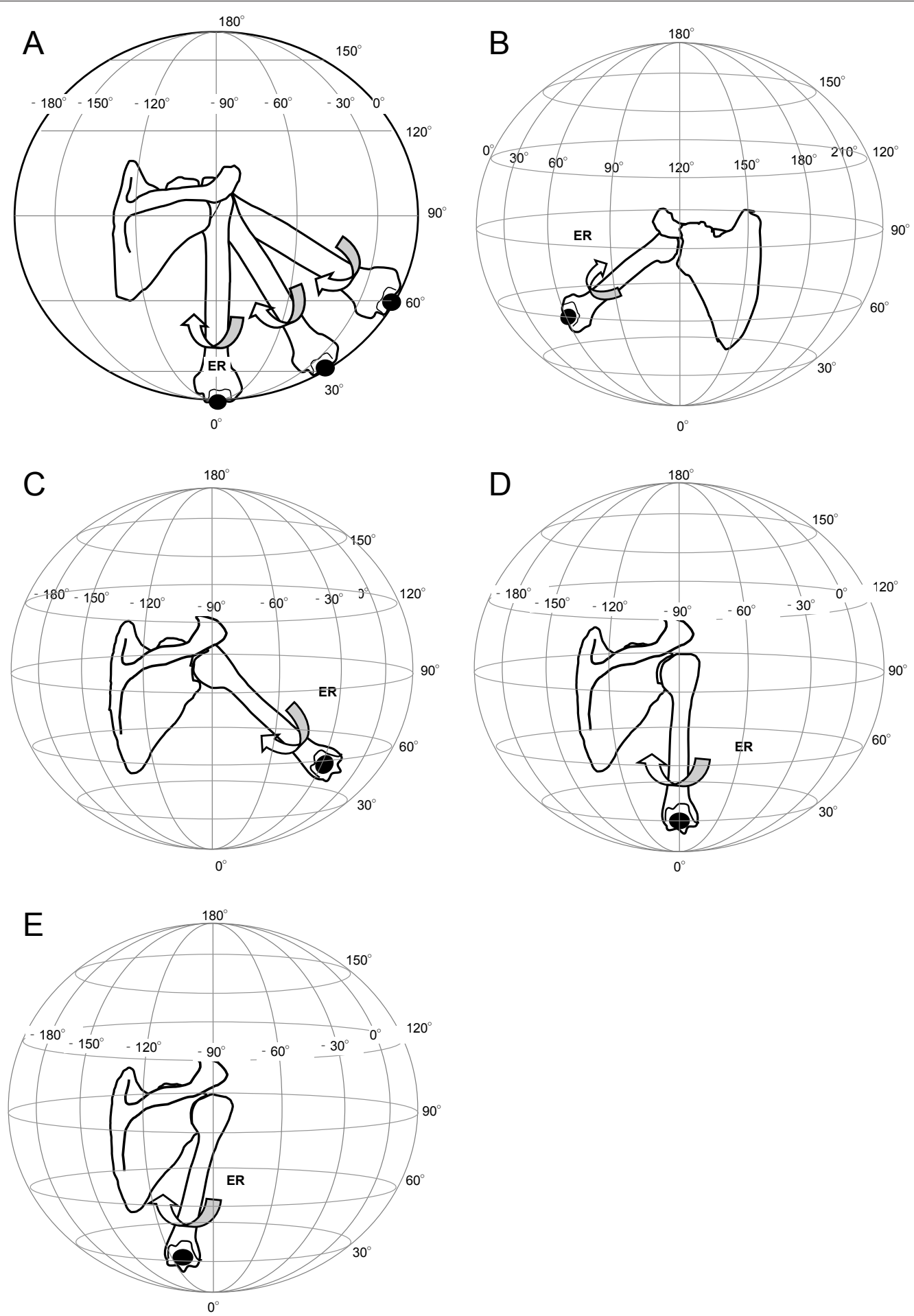

Figure 3 Measurement positions for the glenohumeral joint in vitro. External rotation at $0^{\circ}, 30^{\circ}$ and $60^{\circ}$ elevation in the scapula plane (Figure 3-A), external rotation at $30^{\circ}$ and $60^{\circ}$ flexion (Figure 3-B), external rotation at $30^{\circ}$ and $60^{\circ}$ abduction (Figure $3-C$ ), external rotation at $30^{\circ}$ extension (Figure 3-D), external rotation and adduction at $30^{\circ}$ extension (Figure 3-E). In the globe system, the scapula plane was consistent with 0 degree of longitude. Then, the latitude of the globe system indicates elevation angle of the glenohumeral joint. The longitude of globe system indicates horizontal adduction or abduction angle of the glenohumeral joint. Arrows indicated direction of motion of the glenohumeral joint as external rotation. ER: External Rotaion of the glenohumeral joint. 
degrees in frontal plane toward the rib cage in vivo. In this study, we simulated movement of the glenohumeral joint by fixing the ventral aspect of the scapula with a jig. Flexion and abduction and extension were simulated by 60, -30 , and -90 degrees of longitude in the Globe system.

To maintain the tensile properties of the coracohumeral ligament and range of motion of the glenohumeral joint, passive range of motion of the glenohumeral joint was applied 10 times at the end range in each stretching position before the experiment. [32]

Strain on the ligament at each shoulder positions was measured until passive motion reached the terminal range of the glenohumeral joint, which was determined by grade III mobilization after Kaltenborn's procedure. [33] In that grading system, grade III mobilization comprises the manual application of force at a point at which the therapist perceived end-feel of the joint and observed no further stretching of the ligament.

Baseline Position Baseline position: The baseline position of the glenohumeral joint was determined as $0^{\circ}$ of elevation with $30^{\circ}$ of external rotation on the scapular plane. [10]

In this study, disarticulated scapula from the thorax was fixed on the jig in the frontal plane. Therefore, elevation was designated as glenohumeral abduction in the scapular plane, abduction was designated as glenohumeral abduction with 30 degrees horizontal abduction, and flexion was designated as glenohumeral abduction with 60 degrees horizontal adduction.

External Rotation with elevation The glenohumeral joint was elevated to $0^{\circ}, 30^{\circ}$ or $60^{\circ}$ on the scapula plane [11,15-17]. In each shoulder position, passive external rotation was applied from $-10^{\circ}$ to maximal rotation in $10^{\circ}$ increments (Figure 3-A).

External Rotation with flexion The glenohumeral joint was elevated to $30^{\circ}$ or $60^{\circ}$ on the scapular plane with an additional $60^{\circ}$ of horizontal adduction. [17] In each shoulder position, passive external rotation was applied from $-10^{\circ}$ to maximal rotation in $10^{\circ}$ increments (Figure 3B).

External Rotation with abduction The glenohumeral joint was elevated to $30^{\circ}$ or $60^{\circ}$ on the scapula plane with an additional $30^{\circ}$ of horizontal abduction. [11,13] In each shoulder position, passive external rotation was applied from $-10^{\circ}$ to maximal rotation in $10^{\circ}$ increments (Figure 3-C).

External Rotation with extension The glenohumeral joint was elevated to $30^{\circ}$ on the scapula plane with an additional $90^{\circ}$ of horizontal abduction. [14] At this shoulder position, passive external rotation was applied from $-10^{\circ}$ to $50^{\circ}$ in maximal rotation in $10^{\circ}$ increments (Figure 3-D).

External Rotation and adduction with extension The glenohumeral joint was elevated to $30^{\circ}$ on the scapula plane with an additional $90^{\circ}$ of horizontal abduction and maximum adduction. [14] At this shoulder position, passive external rotation was applied from $-10^{\circ}$ to maximal rotation in $10^{\circ}$ increments (Figure 3-E).

Differences between in vivo and cadavers The glenohumeral angle differs in the angle of elevation relative to the thorax. In vivo, the scapula does not rotate from 0 to 30 degrees of elevation of the upper extremity, and after the 30 degrees elevation of the upper extremity, the ratio is $1: 1$. The scapula is then laterally rotated 30 degrees when the arm is elevated at 90 degrees. For this reason, 60 degrees of elevation in this experiment corresponds to 90 degrees arm elevation in vivo. Therefore, rotation, adduction and abduction at 60 degrees of elevation in this experiment correspond to rotation at 90 degrees of elevation and horizontal adduction and abduction in vivo, respectively. [12,28]

\section{Identification of reference length (LO) and data analysis}

Based on the method in previous reports using cadaver shoulders, reference length (L0) was determined for the ligament. $[19,24,34]$ Reference length was the length at which the angle-strain curve of the ligament started to indicate a sudden decrease in strain. The displacement of the ligament was then defined as the change in length from that at LO. On the basis of data obtained from preliminary experiments, the L0 was determined the designated ligament positions to eliminate slack in the ligament. To measure strain on the ligament and capsule, it is possible that true strain on these tissues can be obtained by subtraction of the slack in the tissues.(Figure 4).

As the stretching procedure described in Muscle Stretching in Manual Therapy [35] should be applied to the joint for 10 to 12 seconds after passive motion of the glenohumeral joint has reached the end of the range of movement, each position in our study was maintained for more than 10 seconds until no increase or decrease in strain value was observed. $[19,34]$ The measurements were performed 3 times during each stretching procedure, and a representative value was calculated by averaging the values obtained for each stretching procedure.

\section{Statistical Analysis}

Statistical analysis was performed using SPSS for Windows ver. 11.5 J (SPSS Japan Inc., Tokyo, Japan). Measurement values were analyzed by one-way repeated ANOVA using the raw measurement values for each stretching position. Therefore, reference length 0 was confirmed using the raw measurement values of the distance between the needles at the point where an acute change in the strain was determined. A step-by-step increase in external rotation of the glenohumeral joint was then applied to measure ligament strain. Dunnett's 


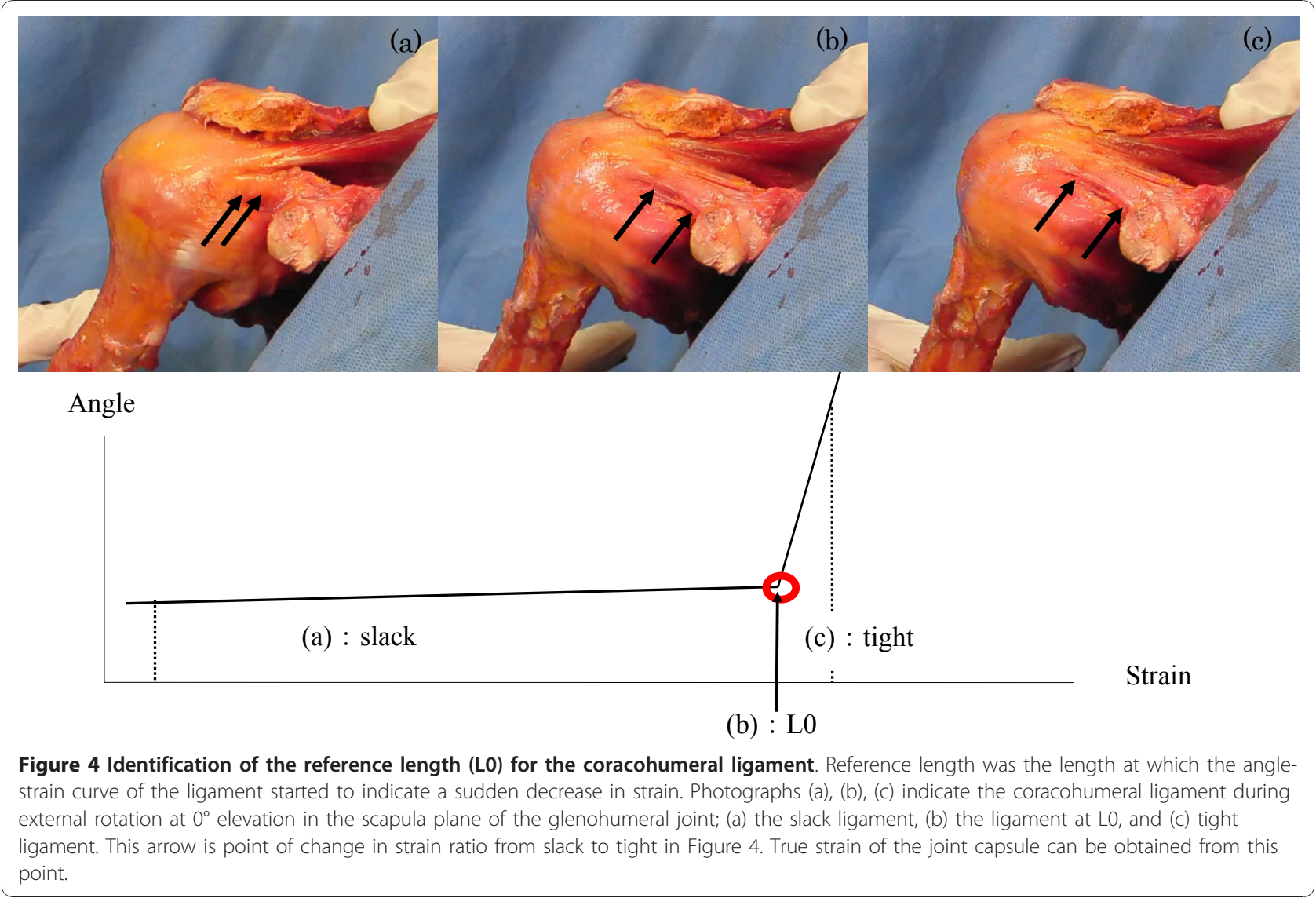

post hoc test was used for multiple comparisons to the raw reference length. The alpha level was set at 0.05 .

Positive strain on each ligament was calculated using the following formula:

$$
\operatorname{Strain}(\%)=\Delta \mathrm{L}(\mathrm{mm}) / \mathrm{L}(\mathrm{mm}) \times 100
$$

Where $\mathrm{L}$ is the length between the points at $\mathrm{L} 0$, and $\Delta \mathrm{L}$ is displacement from L0. Strain values greater than $0 \%$ indicated positive stretching of the ligament from L0. Values less than $0 \%$ indicated no stretching, and are shown as $0 \%$ strain.

\section{Results}

The mean terminal range of passive external rotation at each glenohumeral angle as measured by the 3Space Fastrak system was as follows: $53.8 \pm 5.3^{\circ}, 56.1 \pm 10.4^{\circ}$, $64.6 \pm 22.7^{\circ}$ for $0^{\circ}, 30^{\circ}, 60^{\circ}$ of elevation on the scapula plane, respectively; $58.4 \pm 16.0^{\circ}, 59.9 \pm 15.0^{\circ}$ for $30^{\circ}, 60^{\circ}$ of flexion, respectively; $55.2 \pm 13.4^{\circ}, 57.4 \pm 9.8^{\circ}$ for $30^{\circ}$, $60^{\circ}$ of abduction, respectively; $51.2 \pm 6.8^{\circ}$ for $30^{\circ}$ of extension; $52.1 \pm 13.4^{\circ}$ for $30^{\circ}$ of extension with adduction. The interclass coefficient of the external rotation angles measured by the manual application of maximal external rotation at each measurement positions is 0.877 .
The patterns of increase in strain on the coracohumeral ligament during passive external rotation in various stretching positions are shown in Figure 5. Positive strain on the ligament was obtained from external rotation of greater than $30^{\circ}$ in each shoulder position.

The positive strain on the coracohumeral ligament obtained at each shoulder position is shown in Table 1 . Statistically significant positive strains were obtained at $40^{\circ}, 50^{\circ}$ and maximal external rotation with the arm at $0^{\circ}$ elevation, at $50^{\circ}$ and maximal external rotation with the arm at $30^{\circ}$ extension, and at $30^{\circ}, 40^{\circ}, 50^{\circ}$ and maximal external rotation with arm at $30^{\circ}$ extension with adduction $(P<0.05)$. No positive strain on the coracohumeral ligament was observed by the previously reported stretching positions; i.e., at $90^{\circ}$ abduction with external rotation or during flexion with external rotation.

Figure 6 shown potential in vivo stretching positions for the coracohumeral ligament that correspond to the positions demonstrating significantly increased strain values in vitro.

\section{Discussion}

The coracohumeral ligament is a fibrous structure that contributes to the stability of the glenohumeral joint by 


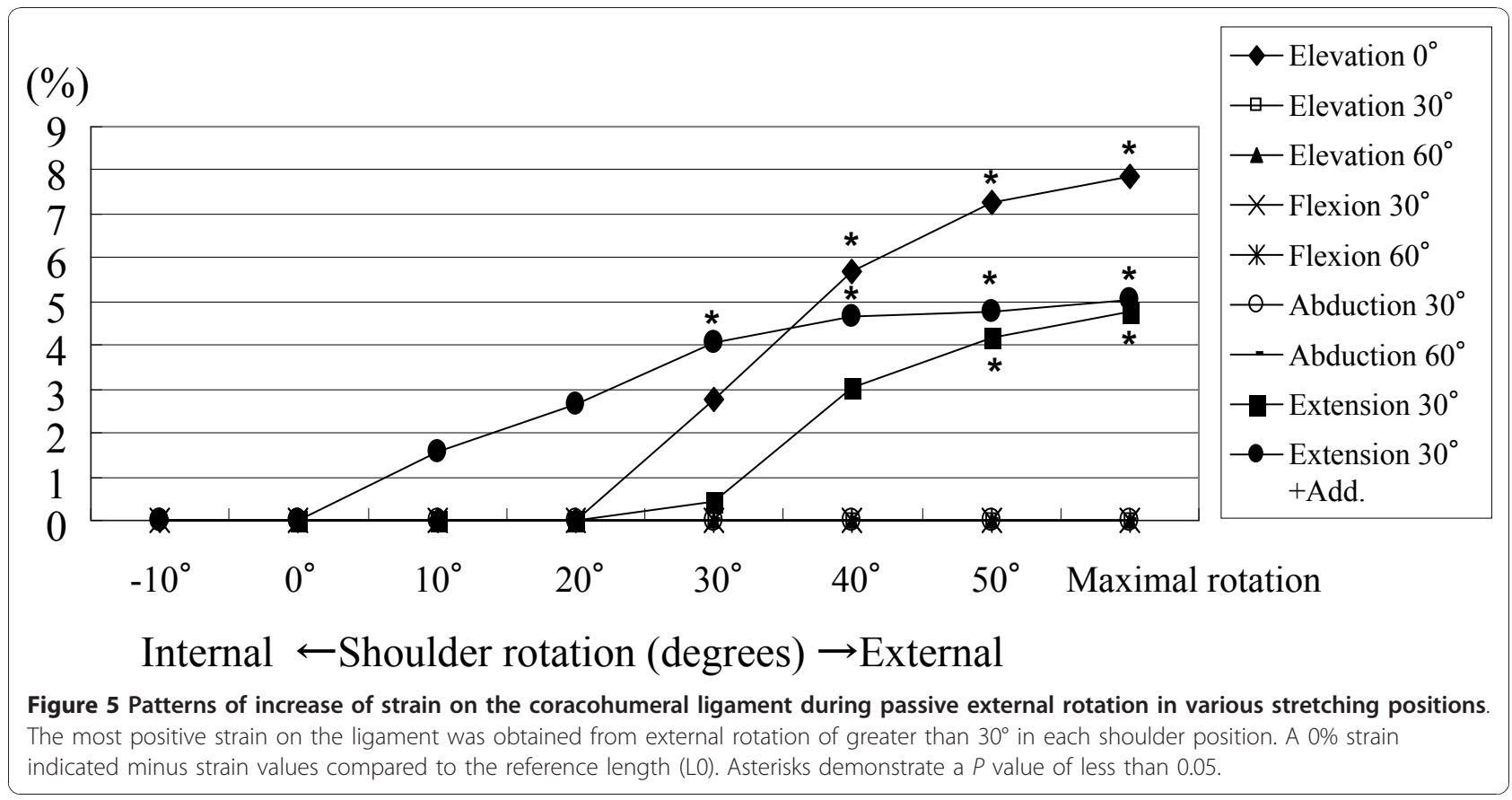

combined action with the rotator cuff muscles, superior glenohumeral ligament, and capsule. In particular, the coracohumeral ligament functions to prevent inferior subluxation of the humeral head, $[13,36]$ thereby playing an important role in the stabilization of the glenohumeral joint in an upright position. In addition, together with the supraspinatus muscle, subscapularis muscle, superior portion of the glenohumeral ligament, and antero-superior portion of the capsule of the glenohumeral joint, the coracohumeral ligament constitutes the rotator interval. Contracture of the rotator interval has been proved to cause contracture of the glenohumeral joint. $[4,26,37]$

Burkart et al [11] reported that the coracohumeral ligament originates from the base of the coracoid process and inserts into both the greater tubercle and lesser tubercle of the humerus. Pouliart et al [25] and Tetro et al [26] reported that the coracohumeral ligament was composed of both superficial and deep fibers, and that the superficial fibers insert into the greater tubercle of the humerus and deep fibers insert into the lesser tubercle. [25,26] Moreover, the fibers inserting into the greater tubercle of the humerus comprise the major fibers of the coracohumeral ligament. [11] In this study, we measured the strain on the superficial fibers of the coracohumeral ligament, which insert into the greater tubercle, using a strain gauge.

According to a previous report on the measurement of the tensile properties of the coracohumeral ligament [38], the cross sectional area of the ligament is five times and maximum load is twice to three times that of the superior fibers of the glenohumeral ligament. Gagey et al [8], Neer et al [5] and Ozaki et al [7] have reported that patients with refractory shoulder contracture were

Table 1 Strain of the Coracohumeral Ligament and Positions of the Glenohumeral Joint

\begin{tabular}{cccc}
\hline Shoulder Positions Measured & Reported Authors & In Vitro Measurement & In Vivo Effective Positioning \\
\hline Elevation $0^{\circ}$ with ER & Burkart & Fig. 3-A & Fig. 7-A, D \\
Elevation $30^{\circ}$ with ER & Kuhn & Fig. 3-A \\
Elevation $60^{\circ}$ with ER & Fig. 3-A \\
Flexion $30^{\circ}$ with ER & Edelson and Ferrari & Fig. 3-B \\
Flexion $60^{\circ}$ with ER & Burkart & Fig. 3-B \\
Abduction $30^{\circ}$ with ER & Edelson and Ferrari & Fig. 3-C \\
Abduction $60^{\circ}$ with ER & Terry and Kelly & Fig. 3-C & Fig. 7-B, E \\
Extension $30^{\circ}$ with ER & Kelly & Fig. 3-D & Fig. 7-C, F \\
\hline
\end{tabular}

Mean of strain \% (standard deviation). $0 \%$ strain indicated minus strain values compared to reference length (L0). Ext, external; Rot, rotation.

*: Statistically significant increase in strain compared to LO; $\mathrm{P}<.05$. 

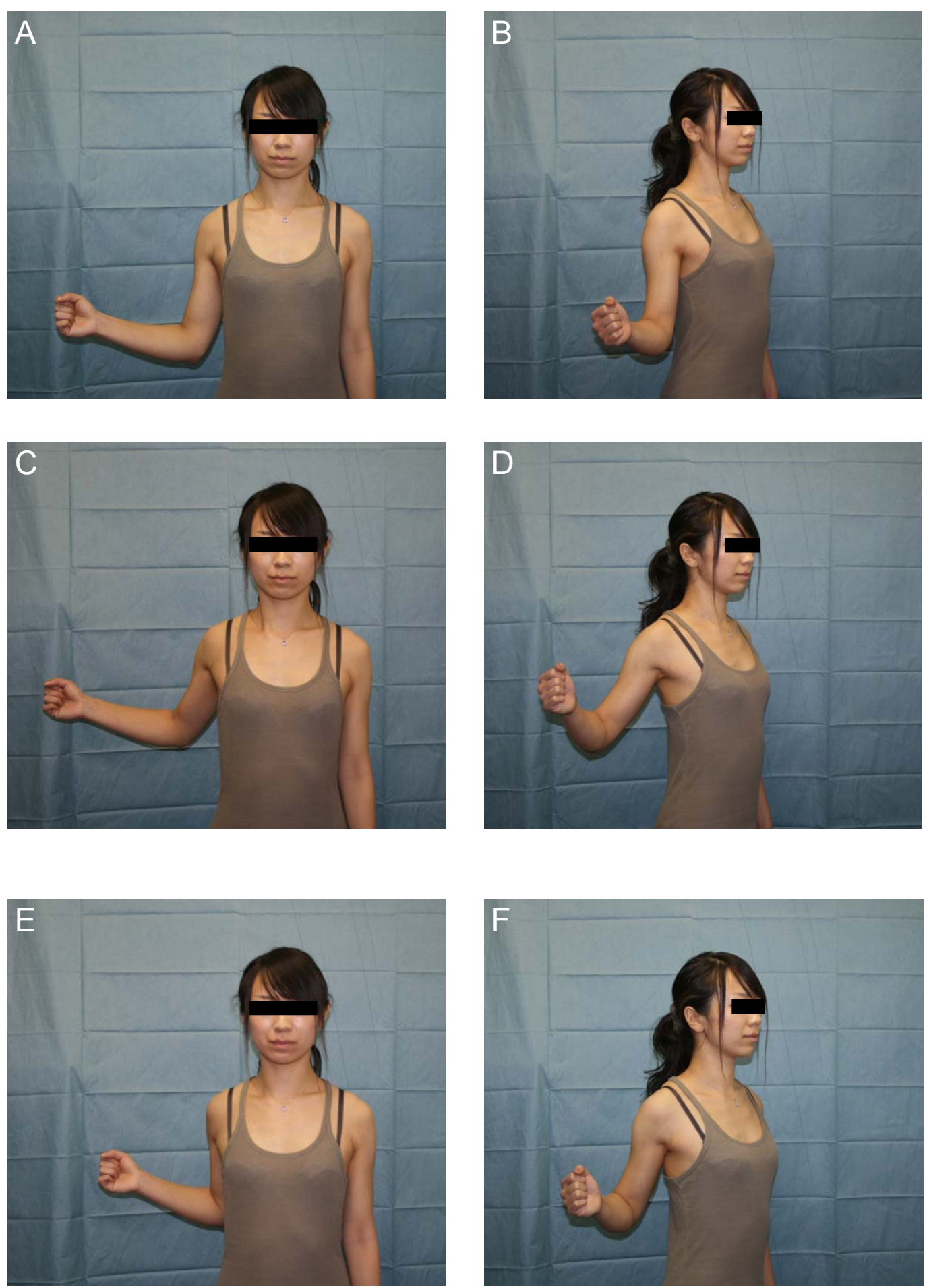

Figure 6 Potential in vivo stretching positions for the coracohumeral ligament that correspond to positions with significantly increased strain values observed in vitro. $A$ and $B, 0$ degrees of elevation with maximum external rotation of the glenohumeral joint. $C$ and $D, 30$ degrees of extension with maximum external rotation of the glenohumeral joint. $E$ and $F, 30$ degrees of extension with adduction with maximum external rotation of the glenohumeral joint. 
successfully treated by surgical resection of the coracohumeral ligament. Therefore, the clarification of effective stretching positions for the coracohumeral ligament is important to treat and prevent shoulder joint contracture by physical treatment.

Various stretching procedures have been reported for the coracohumeral ligament. Burkart et al [11] reported that the coracohumeral ligament restricted the range of motion of external rotation of the glenohumeral joint at 0 degrees of elevation and 50 degrees of abduction. Edelson et al [17] reported that the coracohumeral ligament was tight during flexion and external rotation of the glenohumeral joint, and Ferrari [13] reported that it was tight during external rotation below 60 degrees of abduction. In addition to their studies, Kuhn et al [15] reported that increase in the range of motion of external rotation at 60 degrees of elevation of the glenohumeral joint was recognized when the coracohumeral ligament was released. Terry et al [16] directly measured strain on the coracohumeral ligament in relation to the range of motion of the glenohumeral joint; however, they did not take slack in the ligament into consideration. In their study, flexion and extension of the shoulder joint or external rotation with 0 degrees of elevation were regarded as effective stretching positions for the coracohumeral ligament. Kelley et al [14] reported that positive strain on the coracohumeral ligament was obtained in external rotation with extension, and external rotation with extension plus adduction of the shoulder joint.

In this experiment, a reference length was adopted to measure strain on the coracohumeral ligament. In consequence, positive strain on the coracohumeral ligament was obtained in 9 glenohumeral joint positions; i.e., $40^{\circ}$, $50^{\circ}$ and maximum external rotation at $0^{\circ}$ elevation, $50^{\circ}$ and maximum external rotation at $30^{\circ}$ extension, and $30^{\circ}, 40^{\circ}, 50^{\circ}$ and maximum external rotation at $30^{\circ}$ extension with adduction. These results were consistent with the positions (external rotation at $0^{\circ}$ elevation) reported by Burkart et al [11], Terry et al [16] and
Edelson et al [17]. (Table 2) Moreover, positive strain on the coracohumeral ligament obtained during external rotation with extension, and external rotation with extension and adduction of the shoulder joint was reported by Kelley et al [14]. (Table 2)

The strain on the coracohumeral ligament in this study ranged from $4.20 \%$ to $7.87 \%$. Although these strain values are compatible with those obtained from the lineal region of a stress-strain curve of a tendon or ligament; in this region micro ruptures in the ligament and tendon occur [39], the maximal strain on the inferior glenohumeral ligament was reported by Bigliani et al [29] to be $10.9 \%$. Therefore, stretching of the coracohumeral ligament within the range of $4.20 \%$ to $7.87 \%$ is considered to present little risk of injury to the ligament. Stretching procedures obtained from this mechanical study have the potential to stretch the coracohumeral ligament with safety and are thought to be useful for the treatment of shoulder contracture.

There are several limitations to this study. First, because the specimens were harvested from aged cadavers, the range of motion and mechanical properties of the specimens might be different from those of specimens from younger adults, with the strain on the ligament observed in the aged cadavers likely to be smaller than that on ligament in younger adults. [40]

Second, the interclass coefficient of the measured external rotation angles by the manually application of maximal external rotation at each measurement position was 0.877 in this study, which is good for criterion of interclass coefficient confidence. However, it is true that the rotational torque of the shoulder produced by the manual application using grade III mobilization after Kaltenborn's procedure may fluctuate. We will establish a quantitative measurement system for the applied rotational torque of the shoulder joint using a torque gauge in future studies.

Third, in this study, though humeral head subluxation was carefully observed by three researchers and no

Table 2 In Vitro Positions of the Glenohumeral Joint for the Strain Measurement of Coracohumeral Ligament and Significant Corresponding In Vivo Stretching Positions of the Shoulder

\begin{tabular}{|c|c|c|c|c|c|c|c|c|c|}
\hline & & $-10^{\circ}$ Ext. Rot. & $0^{\circ}$ Ext. Rot. & $10^{\circ}$ Ext. Rot. & $20^{\circ}$ Ext. Rot. & $30^{\circ}$ Ext. Rot. & $40^{\circ}$ Ext. Rot. & $50^{\circ}$ Ext. Rot. & Maximal rotation \\
\hline \multirow[t]{3}{*}{ Elevation } & $0^{\circ}$ & 0 & 0 & 0 & 0 & $2.75(3.17)$ & $5.68(2.16)^{*}$ & $7.27(1.47)^{*}$ & $7.87(1.18)^{*}$ \\
\hline & $30^{\circ}$ & 0 & 0 & 0 & 0 & 0 & 0 & 0 & 0 \\
\hline & $60^{\circ}$ & 0 & 0 & 0 & 0 & 0 & 0 & 0 & 0 \\
\hline \multirow[t]{2}{*}{ Flexion } & $30^{\circ}$ & 0 & 0 & 0 & 0 & 0 & 0 & 0 & 0 \\
\hline & $60^{\circ}$ & 0 & 0 & 0 & 0 & 0 & 0 & 0 & 0 \\
\hline \multirow[t]{2}{*}{ Abduction } & $30^{\circ}$ & 0 & 0 & 0 & 0 & 0 & 0 & 0 & 0 \\
\hline & $60^{\circ}$ & 0 & 0 & 0 & 0 & 0 & 0 & 0 & 0 \\
\hline \multirow[t]{2}{*}{ Extension } & $30^{\circ}$ & 0 & 0 & 0 & 0 & $0.43(2.51)$ & $3.04(1.34)$ & $4.20(1.65)^{*}$ & $4.79(1.67)^{*}$ \\
\hline & $30^{\circ}+$ Add & 0 & 0 & $1.57(3.65)$ & $2.65(3.23)$ & $4.09(3.23)^{*}$ & $4.67(3.40)^{*}$ & $4.78(4.42)^{*}$ & $5.05(5.20)^{*}$ \\
\hline
\end{tabular}

ER, external rotation; Add, Adduction. 
visible and palpable subluxation was detected during experiment, minor translations of the humeral head might have occurred without being noticed.

\section{Conclusions}

Stretching positions of the coracohumeral ligament in which significantly larger strains were obtained were $0^{\circ}$ elevation with $40^{\circ}, 50^{\circ}$ and maximum passive external rotation, $30^{\circ}$ extension with $50^{\circ}$ and maximum external rotation, and $30^{\circ}$ extension + adduction with $30^{\circ}, 40^{\circ}$, $50^{\circ}$ and maximum external rotation. No positive strain was observed on the coracohumeral ligament using previously reported stretching positions; i.e., $90^{\circ}$ abduction with external rotation or flexion with external rotation. Stretching procedures obtained from this in vitro mechanical study have the potential to stretch the coracohumeral ligament with considerable safety in vivo and are thought to be useful for the treatment of shoulder contracture.

\section{Acknowledgements}

We also thank Masaki Katayose PT, PhD, Egi Hidaka PT, MS and Misaki Fujii PT, MS, for their technical support and useful suggestion.

\section{Author details}

'Doctoral Course of Physical Therapy, Graduate School of Health Sciences, Sapporo Medical University, Sapporo, Japan. ${ }^{2}$ Department of Orthopaedic Surgery, Sapporo Daiichi Hospital, Sapporo, Japan. ${ }^{3}$ Department of Orthopaedic Surgery, Japan Self Defence Force Sapporo General Hospital, Sapporo, Japan. ${ }^{4}$ Department of Physical Therapy, Sapporo Medical University, Sapporo, Japan. ${ }^{5}$ 2nd Department of Anatomy, Sapporo Medical University, Sapporo, Japan. ${ }^{6}$ Department of Physical Therapy, Hokkaido Bunkyo University, Eniwa, Japan.

\section{Authors' contributions}

T I and M A provided concept/idea/research design. TI provided writing and data analysis. TI and Y T provided data collection. E U provided project management. S M provided facilities/equipment and consultation. M F and D S managed of fresh/frozen cadaver specimens. All authors read and approved the final manuscript.

\section{Competing interests}

The authors declare that they have no competing interests.

Received: 7 July 2010 Accepted: 19 January 2011

Published: 19 January 2011

\section{References}

1. Rundquist PJ, Anderson DD, Guanche CA, Ludewig PM: Shoulder kinematics in subjects with frozen shoulder. Arch Phys Med Rehabil 2003, 84:1473-1479.

2. Tauro JC: Stiffness and rotator cuff tears: incidence, arthroscopic findings, and treatment results. Arthroscopy 2006, 22:581-586.

3. Maitland GD: Manipulation: Definition and role. Peripheral manipulation. 3 edition. London: Butterworth-Heinemann; 1991, 9-12

4. Harryman DT, Sidles JA, Harris SL, Matsen FA: The role of the rotator interval capsule in passive motion and stability of the shoulder. J Bone Joint Surg Am 1992, 74:53-66.

5. Neer CS, Satterlee CC, Dalsey RM, Flatow EL: The anatomy and potential effects of contracture of the coracohumeral ligament. Clin Orthop Relat Res 1992, 182-185.

6. Omari A, Bunker TD: Open surgical release for frozen shoulder: surgical findings and results of the release. J Shoulder Elbow Surg 2001, 10:353-357.
7. Ozaki J, Nakagawa Y, Sakurai G, Tamai S: Recalcitrant chronic adhesive capsulitis of the shoulder. Role of contracture of the coracohumeral ligament and rotator interval in pathogenesis and treatment. J Bone Joint Surg Am 1989, 71:1511-1515.

8. Gagey OJ, Boisrenoult P: Shoulder capsule shrinkage and consequences on shoulder movements. Clin Orthop Relat Res 2004, 218-222.

9. Bowen MK, Warren RF: Ligamentous control of shoulder stability based on selective cutting and static translation experiments. Clin Sports Med 1991, 10:757-782.

10. Warner JJ, Deng XH, Warren RF, Torzilli PA: Static capsuloligamentous restraints to superior-inferior translation of the glenohumeral joint. Am J Sports Med 1992, 20:675-685.

11. Burkart AC, Debski RE: Anatomy and function of the glenohumeral ligaments in anterior shoulder instability. Clin Orthop Relat Res 2002, 32-39.

12. Culham E, Peat M: Functional anatomy of the shoulder complex. J Orthop Sports Phys Ther 1993, 18:342-350.

13. Ferrari DA: Capsular ligaments of the shoulder. Anatomical and functional study of the anterior superior capsule. Am J Sports Med 1990, 18:20-24.

14. Kelley MJ, McClure PW, Leggin BG: Frozen shoulder: evidence and a proposed model guiding rehabilitation. J Orthop Sports Phys Ther 2009, 39:135-148.

15. Kuhn JE, Bey MJ, Huston LJ, Blasier RB, Soslowsky LJ: Ligamentous restraints to external rotation of the humerus in the late-cocking phase of throwing. A cadaveric biomechanical investigation. Am J Sports Med 2000, 28:200-205.

16. Terry GC, Hammon D, France $P$, Norwood LA: The stabilizing function of passive shoulder restraints. Am J Sports Med 1991, 19:26-34.

17. Edelson JG, Taitz C, Grishkan A: The coracohumeral ligament. Anatomy of a substantial but neglected structure. J Bone Joint Surg Br 1991, 73:150-153.

18. Leffert RD, Rowe CR: Tendon ruptures. In The Shoulder. Volume 3. Edited by: Rowe CR. London: Butterworth-Heinemann; 1988:131-154.

19. Izumi T, Aoki M, Muraki T, Hidaka E, Miyamoto S: Stretching positions for the posterior capsule of the glenohumeral joint: strain measurement using cadaver specimens. Am J Sports Med 2008, 36:2014-2022.

20. Muraki T, Aoki M, Uchiyama E, Murakami G, Miyamoto S: The effect of arm position on stretching of the supraspinatus, infraspinatus, and posterior portion of deltoid muscles: a cadaveric study. Clin Biomech (Bristol, Avon) 2006, 21:474-480.

21. Muraki T, Aoki M, Uchiyama E, Takasaki H, Murakami G, Miyamoto S: A cadaveric study of strain on the subscapularis muscle. Arch Phys Med Rehabil 2007, 88:941-946.

22. Tibone JE, McMahon PJ, Shrader TA, Sandusky MD, Lee TQ: Glenohumeral joint translation after arthroscopic, nonablative, thermal capsuloplasty with a laser. Am J Sports Med 1998, 26:495-498.

23. Hidaka E, Aoki M, Muraki T, Izumi T, Fujii M, Miyamoto S: Evaluation of stretching position by measurement of strain on the ilio-femoral ligaments: an in vitro simulation using trans-lumbar cadaver specimens. Man Ther 2009, 14:427-432.

24. Ozeki S, Yasuda K, Kaneda K, Yamakoshi K, Yamanoi T: Simultaneous strain measurement with determination of a zero strain reference for the medial and lateral ligaments of the ankle. Foot Ankle Int 2002, 23:825-832.

25. Pouliart N, Somers K, Eid S, Gagey O: Variations in the superior capsuloligamentous complex and description of a new ligament. J Shoulder Elbow Surg 2007, 16:821-836.

26. Tetro AM, Bauer G, Hollstien SB, Yamaguchi K: Arthroscopic release of the rotator interval and coracohumeral ligament: An anatomic study in cadavers. Arthroscopy 2002, 18:145-150.

27. 3SPACE FASTRAK User's Manual. Colchester: Polhemus Inc; 2002, Revision F edition.

28. Halder AM, Itoi $\mathrm{E}$, An KN: Anatomy and biomechanics of the shoulder. Orthop Clin North Am 2000, 31:159-176.

29. Bigliani LU, Pollock RG, Soslowsky LJ, Flatow EL, Pawluk RJ, Mow VC: Tensile properties of the inferior glenohumeral ligament. J Orthop Res 1992 10:187-197.

30. Noyes FR, Butler DL, Grood ES, Zernicke RF, Hefzy MS: Biomechanical analysis of human ligament grafts used in knee-ligament repairs and reconstructions. J Bone Joint Surg Am 1984, 66:344-352.

31. Doorenbosch CA, Harlaar J, Veeger DH: The globe system: an unambiguous description of shoulder positions in daily life movements. J Rehabil Res Dev 2003, 40:147-155. 
32. Van Ee CA, Chasse AL, Myers BS: Quantifying skeletal muscle properties in cadaveric test specimens: effects of mechanical loading, postmortem time, and freezer storage. J Biomech Eng 2000, 122:9-14.

33. Kaltenborn FM: Translatoric joint play. Manual mobilzation of the joints: The Kaltenborn method of joint examination and treatment Vol 1 The Extremity. 5 edition. Oslo: Olaf Norlis bokhandel; 1999, 21-28, Volume The extremity.

34. Urayama M, Itoi E, Hatakeyama Y, Pradhan RL, Sato K: Function of the 3 portions of the inferior glenohumeral ligament: a cadaveric study. J Shoulder Elbow Surg 2001, 10:589-594.

35. Evjenth O, Hamberg J: General principles of relaxation and stretching of muscles and other structures. In Musule stretching in manual therapy: A clinical manual Voll The Extremities. Edited by: Evjenth O, Hamberg J. Alfta: Alfta rehab; 1984:7-12, Volume The Extremities.

36. Fitzpatrick MJ, Tibone JE, Grossman M, McGarry MH, Lee TQ: Development of cadaveric models of a thrower's shoulder. J Shoulder Elbow Surg 2005, 14:49S-57S.

37. Hunt SA, Kwon YW, Zuckerman JD: The rotator interval: anatomy, pathology, and strategies for treatment. J Am Acad Orthop Surg 2007, 15:218-227.

38. Boardman ND, Debski RE, Warner JJ, Taskiran E, Maddox L, Imhoff AB, Fu FH, Woo SL: Tensile properties of the superior glenohumeral and coracohumeral ligaments. J Shoulder Elbow Surg 1996, 5:249-254.

39. Tillman LJ, Chansan NP: Properties of dense connective tissue and wound healing. In Management of common musculoskeltal disorders: Physical therapy principles and methods. Edited by: Hertling D, Randolph M. Philadelphia: J.B. Lippincott; 1996:8-21, Volume 3rd.

40. Yamada H: Compaison of the mechanical properties of human organs and tissues. In Strength of Biological Materials. Edited by: Evans FG. Baltimore: Williams 1970:248-280

doi:10.1186/1758-2555-3-2

Cite this article as: Izumi et al: Stretching positions for the coracohumeral ligament: Strain measurement during passive motion using fresh/frozen cadaver shoulders. Sports Medicine, Arthroscopy, Rehabilitation, Therapy \& Technology 2011 3:2.

\section{Submit your next manuscript to BioMed Central} and take full advantage of:

- Convenient online submission

- Thorough peer review

- No space constraints or color figure charges

- Immediate publication on acceptance

- Inclusion in PubMed, CAS, Scopus and Google Scholar

- Research which is freely available for redistribution

Submit your manuscript at www.biomedcentral.com/submit
Biomed Central 